\title{
PROTEOMIC IMPLICATIONS OF TUMORAL INFILTRATING LYMPHOCYTES IN MELANOMA: PD-L1, CD4 AND GD8 - SHORT REVIEW
}

\section{Nicolau Anca Antonela1,2, Caraban Bogdan Marian',2, Gheorghe Emma1,3, Hangan Tony Laurentiu, ${ }^{13}$, Cojocaru Oana ${ }^{1,3}$, Orasanu Gristian Ionut ${ }^{1}$, , Voda Raluca Ioana ${ }^{1,2}$}

${ }^{1}$ County Clinical Emergency Hospital of Constanta, Romania

${ }^{2}$ Center for Research and Development of the Morphological and Genetic Studies of Malignant Pathology, "Ovidius" University of Constanta

${ }^{3}$ Faculty of Medicine, University "Ovidius" of Contanta

\begin{abstract}
Melanoma is a tumor developed by malignancy of melanocytes, being one of the most lethal cancers. Usually, it is associated with exposure to ultraviolet radiations, being most common in the skin, but can also be located extracutaneously as in the digestive tract, leptomeninges or uvea. Histopathologically it presents a phase of radial growth and a vertical one, often accompanied by an intra and peritumoral inflammatory infiltrate. Immunohistochemically, the confirmation of the diagnosis of melanoma should be accompanied by the assessment of proteomic markers of lymphocytic infiltrate such as PD-L1,CD4 and CD8. Those have a role in evaluating the prognosis and a possible prediction of the immunotherapeutic response.
\end{abstract}

Keywords: CD4, CD8, immunohistochemistry, melanoma, PD-L1.

\section{Introduction}

Of all skin cancers, melanoma is the most fatal, originating in melanocytes, the most common form being skin melanoma. Given the high levels of somatic mutations and antigenspecific tumor $\mathrm{T}$ cells, melanoma is considered an immunogenic tumor. These aspects have made immunotherapy an option for these patients (1).

An essential histopathological parameter in the diagnosis of melanocytic tumors is represented by the tumoral infiltrating lymphocytes (TIL). It is composed of regulatory $\mathrm{T}$ lymphocytes, effector $\mathrm{T}$ lymphocytes, natural killer lymphocytes, macrophages and dendritic cells (2). Depending on the histopathological aspect, it is divided into absent, non-brisk or brisk (3). Absent TIL is defined when it is not identified or is present but does not infiltrate. Non-brisk TIL present focally or in several areas during the vertical growth phase and is associated with an unfavorable prognosis $(3,4)$. TIL brisk is present in the tumor within the vertical growth phase or when it is present and infiltrates the base of the tumor in this phase (3). Thus, the TIL aspect represents in itself a prognostic marker and indicates the strength of the organism's immune system response (4).

Therefore, the need for immunohistochemistry is required to identify 
and quantify some very important markers (PD-L1, CD4 and CD8) in assessing both the prognosis and the survival rate and in assessing the response to certain therapies $(5,6)$.

\section{Discussion}

PD-L1 is a PD-1 ligand, being expressed by T lymphocytes, B lymphocytes, dendritic cells, macrophages, placental trophoblasts, epithelial cells of the thymic cortex and myocardial endothelium $(7,8)$. It can also be identified in tumors such as melanoma, renal cell carcinoma, gastric cancer, non-small cell lung cancer, breast cancer (9). PD-1 is produced mostly by activated $\mathrm{T}$ lymphocytes and memory cells; in malignant melanoma, it is acting as a promoter of tumorogenesis. The role of PD-L1 intervenes in the activity of $\mathrm{T}$ lymphocytes by downregulation of the receptor T-cell, thus preventing the hyperactivation of T lymphocytes (7).

The mechanism of action begins with tumor recognition by the tumor lymphocyte infiltrate (TIL), that produces cytokines and gamma interferon (IFN). This secretion increases the expression of PD-L1. The interaction with PD-1 will form the PD-L1/PD-1 complex which will cause down-modulation of the tumor lymphocyte infiltrate (10). The consequences are inhibition of $\mathrm{T}$ cell growth and cytokine production (9). Situations are described when PD-L1 is expressed in the absence of TIL and vice-versa the lymphocytic infiltrate can exist without the expression of PD-L1 (7). This aspect led to the division of the tumor microenvironment into four types: Type I (PD-L1+/ TIL+) 38\%, Type II (PD-L1-/TIL-) 41\%, Type III (PD-L1+/TIL-) $1 \%$ and Type IV (PD-L1-/TIL+) 20\% (6). PD-L1 expression increases with disease progression, being found in a higher percentage in melanoma metastases than in primary melanomas (9).

Immunohistochemically, PD-L1 is found on the surface of tumor cell membranes and inflammatory cells. It is difficult to accurately quantify the cut-off value, because each evaluator can establish his own working method. Usually a cut-off of $>25 \%$ in tumor cells and $>5 \%$ in cell infiltrate are used $(11,12)$.

It should be noted that overexpression causes the tumor cells to become immune to in vitro cell lysis exerted by CD8 T lymphocytes (13). According to the study by Kaunitz GJ et al. the melanoma with the lowest presence of PD-L1+ is the uveal one ( $10 \%$ of cases), and cumulative solar damage melanoma has the highest proportion of PD-L1 positive $(62 \%$ of cases). This denotes a variety of response depending on of each subtype of melanoma (10).

A negative expression correlates with a short survival and poor response to treatment, in this sense it can be used as a prognostic marker (7). Patients with PD-L1 positive expression have a very good response and an increased expectation of survival by using treatments based on inhibition of the Mitogen-activated protein kinase (MAPK) pathway (14). Their high rate of response to treatment does not disregard the therapy of PD-L1 negative, which of course can benefit from the same treatment, but with a lower effectiveness (13).

Regarding the mediators of antitumor immunity, $\mathrm{T}$ lymphocytes have a role of maximum importance by associating the action of CD4 T cells and cytotoxic CD8 T lymphocytes (15).

CD4 T cells come from the differentiation of naive cells on the JAK/STAT pathway, in which transcription factors, hormone receptors and nuclear regulators participate, having as main role the immune protection through the production of gamma-IFN and IL-4 (16).

The mechanism of tumor antigen recognition is achieved by the association of CD4 lymphocytes with HLA II molecules. These molecules are found on the surface of activated B lymphocytes, macrophages and dendritic cells. They come in contact with tumor antigens, which they will later phagocytose in order to process and activate T-helper cells (15).

Frequent, the recognition of malignant cells directly by CD4 T lymphocytes will not be possible secondary to the absence of the major histocompatibility complex II (MHC II) in the structure of solid malignancies. Despite this feature, in more than $40 \%$ of melanomas the molecules of the complex may be an integral part of the tumor or may appear as a result of stimulation by gamma-IFN (5). In melanoma, the constituent of lymphocytic tumor infiltrate 
are CD4 $\mathrm{T}$ lymphocytes specific for restricted tumor antigens class II (17-19). These cells can mainly have an anticancer effect by helping CD8 + T lymphocytes or by directly recognizing the antigens present on the cell surface (5).

In addition to the support given to $\mathrm{CD} 8$ cytotoxic lymphocytes, CD4 + T cells may have a direct cytotoxic effect on malignant melanocytes, and studies have been reported showing the intervention of CD4 $\mathrm{T}$ lymphocytes in vitro tumors lysis by the interaction of the target cell with the APO-1 (20-22).

CD4 $\mathrm{T}$ cells together with their activator markers, such as CD25 or CD134, have a favorable prognostic role in melanoma, when they are identified in an increased peritumoral density, but not when they are identified within the tumor (23).

Most of the effector cells are cytotoxic CD8 lymphocytes, whose lytic effect is achieved by perforin/granzyme released from the granules they contain $(15,20)$.

Activated CD8 $+\mathrm{T}$ cells will induce cell death via the perforin-granzyme ligand and Fas/Fas ligand pathways. The major role of $\mathrm{CD} 8+\mathrm{T}$ cells is in the secretion of gammaIFN responsible for an up-regulating $\mathrm{MCH}$ I and II, acting directly in the differentiation of CD4+ Th cells (24). Ferroptosis is a form of cell death resulting from the accumulation of iron-dependent lipid peroxides $(25,26)$. In the study conducted by Wang W et al, they observed that immunotherapy-activated $\mathrm{CD} 8+\mathrm{T}$ cells improved lipid peroxidation in malignant cells, so that the amplification of ferroptosis contributes to the effectiveness of immunotherapy (27).

Activated CD8+ cells have on the cell surface CD152 (CTLA-4), having a role in inhibiting the immune response. Ipilimumabbased therapies (Anti-CTLA-4 antibody) show an early increase in the absolute number of lymphocytes that occurred within 2-8 weeks after the first cycle of treatment. This increase is correlated with the improvement of survival $(28,29)$. The increase in the absolute number of lymphocytes is achieved based on the increase of density of both CD8+ and CD4+ cells (29). Early increase in the absolute number of lymphocytes, associated with the increase in the number of $\mathrm{CD} 4+$ and $\mathrm{CD} 8+$ cells also occurs later, after 8-14 weeks. From the point of view of cancer-directed immunotherapy, it restores and improves the action of CD8+ lymphocytes in the tumor (27). The two events mentioned above indicate a change in the immune system, which may highlight the transition from a nonspecific immunity to a specific type (29).

According to the results obtained by Piras $F$ et al, depending on the density of the cells arranged adjacent to the normal dermis, at the base of melanomas with vertical growth phase, the mortality rate varied from $81 \%$ in cases with low density to $20 \%$ in cases with a high density of lymphocytes CD8+. The 5-year survival rate in low-density cases was $25 \%$ and $78.8 \%$ in high-density lymphocyte cases. Following these results there is a statistically significant correlation between CD8 cell count and survival (15).

Quantification of membrane immunoexpression of CD4 and CD8 markers does not present standard assessment criteria, this being a decision of the pathologist's working protocol (manual, semi-automatic or automatic), the only condition involving such a standardized system is colon cancer $(30,31)$.

\section{Conclusion}

Malignant melanoma is an immunogenic tumor that associates an inflammatory infiltrate consisting of regulatory $\mathrm{T}$ lymphocytes, effector $\mathrm{T}$ lymphocytes, natural killer lymphocytes, macrophages and dendritic cells, whose particularly important markers are represented by CD4, CD8 and PD-L1. Even if they mainly have different mechanisms of action, they are focally entangled by gamma-IFN, which stimulates the production of lymphocytes and PD-L1, the second one will reduce the number of peritumoral lymphocytes by feed-back mechanism.

\section{Acknowledgements}

This work was supported by "Ovidius" University of Constanţa through the grant no. 6/14.11.2018

For this article all the authors have equal contributions. 


\section{Conflict of interest}

The authors declare that there is no conflict of interest.

\section{References}

1. Rad Pour S, Morikawa H, Kiani NA, Yang M, Azimi A, Shafi G, et al. Exhaustion of CD4+ T-cells mediated by the Kynurenine Pathway in Melanoma. Sci Rep. 2019;9(1):12150.

2. Antohe M, Nedelcu RI, Nichita L, Popp CG, Cioplea M, Brinzea A, et al. Tumor infiltrating lymphocytes: The regulator of melanoma evolution. Oncol Lett. 2019 May;17(5):4155-61.

3. Hendry S, Salgado R, Gevaert T, Russell PA, John T, Thapa B, et al. Assessing TumorInfiltrating Lymphocytes in Solid Tumors: A Practical Review for Pathologists and Proposal for a Standardized Method from the International Immuno-Oncology Biomarkers Working Group: Part 2: TILs in Melanoma, Gastrointestinal Tract Carcinomas, Non-Small Cell Lung Carcinoma and Mesothelioma, Endometrial and Ovarian Carcinomas, Squamous Cell Carcinoma of the Head and Neck, Genitourinary Carcinomas, and Primary Brain Tumors. Adv Anat Pathol. 2017 Nov;24(6):311-35.

4. Weiss SA, Han SW, LuiK, TchackJ, Shapiro R, Berman R, et al. Immunologic heterogeneity of tumor-infiltrating lymphocyte composition in primary melanoma. Hum Pathol. 2016 Nov;57:116-25.

5. Hadrup S, Donia M, Thor Straten P. Effector CD4 and CD8 T cells and their role in the tumor microenvironment. Cancer Microenviron. 2013 Aug;6(2):123-33.

6. Teng MW, Ngiow SF, Ribas A, Smyth MJ. Classifying Cancers Based on T-cell Infiltration and PD-L1. Cancer Res. 2015;75(11):2139-2145.

7. Mandalà M, Merelli B, Massi D. PD-L1 in melanoma: facts and myths. Melanoma Manag. 2016;3(3):187-194.

8. Obeid JM, Erdag G, Smolkin ME, et al. PD-L1, PD-L2 and PD-1 expression in metastatic melanoma: Correlation with tumor-infiltrating immune cells and clinical outcome. Oncoimmunology. 2016;5(11):e1235107.

9. Gadiot J, Hooijkaas AI, Kaiser AD, van Tinteren $H$, van Boven $H$, Blank C. Overall survival and PD-L1 expression in metastasized malignant melanoma. Cancer. 2011;117(10):2192-2201.

10. Kaunitz GJ, Cottrell TR, Lilo $M$, et al. Melanoma subtypes demonstrate distinct PD-L1 expression profiles. Lab Invest. 2017;97(9):1063-1071.

11. Hirsch FR, McElhinny A, Stanforth D, et al. PD-L1 Immunohistochemistry Assays for Lung Cancer: Results from Phase 1 of the Blueprint PD-L1 IHC Assay Comparison Project. J Thorac Oncol. 2017;12(2):208222.

12. Zajac M,YeJ, Mukhopadhyay P, etal. Optimal PD-L1-high cutoff for association with overall survival in patients with urothelial cancer treated with durvalumab monotherapy. PLoS One. 2020;15(4):e0231936.

13. Mahoney KM, Freeman GJ, McDermott DF. The Next Immune-Checkpoint Inhibitors: PD-1/PD-L1 Blockade in Melanoma. Clin Ther. 2015;37(4):764-782.

14. Kakavand H, Rawson RV, Pupo GM, et al. PD-L1 Expression and Immune Escape in Melanoma Resistance to MAPK Inhibitors. Clin Cancer Res. 2017;23(20):6054-6061.

15. Piras F, Colombari R, Minerba L, et al. The predictive value of CD8, CD4, CD68, and human leukocyte antigen-D-related cells in the prognosis of cutaneous malignant melanoma with vertical growth phase. Cancer. 2005;104(6):1246-1254.

16. Zhu J, Paul WE. CD4 T cells: fates, functions, and faults. Blood. 2008;112(5):1557-1569.

17. Dadmarz R, Sgagias MK, Rosenberg SA, Schwartzentruber DJ. CD4+ T lymphocytes infiltrating human breast cancer recognise autologous tumor in an MHC-class-II restricted fashion [published correction appears in Cancer Immunol Immunother 1995 Sep;41(3):201]. Cancer Immunol Immunother. 1995;40(1):1-9.

18. Dadmarz RD, Ordoubadi A, Mixon A, et al. Tumor-infiltrating lymphocytes from human ovarian cancer patients recognize autologous 
tumor in an MHC class II-restricted fashion. Cancer J Sci Am. 1996;2(5):263-272.

19. Robbins PF, El-Gamil M, Li YF, Zeng G, Dudley M, Rosenberg SA. Multiple HLA class II-restricted melanocyte differentiation antigens are recognized by tumor-infiltrating lymphocytes from a patient with melanoma. J Immunol. 2002;169(10):6036-6047.

20. Thomas WD, Hersey P. CD4 T cells kill melanoma cells by mechanisms that are independent of Fas (CD95). Int J Cancer. 1998;75(3):384-390.

21. Chen Q, Hersey P. MHC-restricted responses of $\mathrm{CD} 8+$ and $\mathrm{CD} 4+\mathrm{T}$-cell clones from regional lymph nodes of melanoma patients. Int J Cancer. 1992;51(2):218-224.

22. Takahashi T, Chapman PB, Yang SY, Hara I, Vijayasaradhi S, Houghton AN. Reactivity of autologous CD4+ T lymphocytes against human melanoma. J Immunol. 1995;154:772779.

23. Ladányi A. Prognostic and predictive significance of immune cells infiltrating cutaneous melanoma. Pigment Cell Melanoma Res. 2015;28(5):490-500.

24. Pandiyan P, Hegel JK, Krueger M, Quandt D, Brunner-Weinzierl MC. High IFN-gamma production of individual CD8 T lymphocytes is controlled by CD152 (CTLA-4). J Immunol. 2007;178(4):2132-2140.

25. Dixon SJ, Lemberg KM, Lamprecht MR, et al. Ferroptosis: an iron-dependent form of nonapoptotic cell death. Cell. 2012;149(5):1060-1072.

26. Yang WS, SriRamaratnam R, Welsch ME, et al. Regulation of ferroptotic cancer cell death by GPX4. Cell. 2014;156(1-2):317-331.

27. Wang W, Green M, Choi JE, et al. CD8+ $\mathrm{T}$ cells regulate tumour ferroptosis during cancer immunotherapy. Nature. 2019;569(7755):270-274.

28. Cameron F, Whiteside G, Perry C. Ipilimumab: first global approval. Drugs. 2011;71(8):1093-1104.

29. Martens A, Wistuba-Hamprecht K, Yuan J, et al. Increases in Absolute Lymphocytes and Circulating CD4+ and CD8+ T Cells Are Associated with Positive Clinical Outcome of Melanoma Patients Treated with Ipilimumab. Clin Cancer Res. 2016;22(19):4848-4858.
30. Maibach F, Sadozai H, Seyed Jafari SM, Hunger RE, Schenk M. Tumor-Infiltrating Lymphocytes and Their Prognostic Value in Cutaneous Melanoma. Front Immunol. 2020;11:2105.

31. Steele KE, Tan TH, Korn R, et al. Measuring multiple parameters of $\mathrm{CD} 8+$ tumorinfiltrating lymphocytes in human cancers by image analysis. J Immunother Cancer. 2018;6(1):20. 